\title{
A comprehensive assessment of the prognostic role of cell adhesion molecules in acute myeloid leukemia
}

\author{
Jing Cheng ${ }^{1 \#}$, Juan $\operatorname{Han}^{2 \#}$, Chunyan Lin ${ }^{1}$ \\ ${ }^{1}$ Department of Blood Transfusion, The First Affiliated Hospital of Soochow University, Suzhou, China; ${ }^{2}$ Department of Laboratory, 904th Hospital \\ of Joint Logistic Support Force of PLA, Suzhou, China \\ Contributions: (I) Conception and design: C Lin; (II) Administrative support: C Lin; (III) Provision of study materials or patients: J Cheng; (IV) \\ Collection and assembly of data: J Cheng, J Han; (V) Data analysis and interpretation: J Han; (VI) Manuscript writing: All authors; (VII) Final \\ approval of manuscript: All authors. \\ "These authors contributed equally to this work and should be considered as co-first authors. \\ Correspondence to: Chunyan Lin. Department of Blood Transfusion, The First Affiliated Hospital of Suzhou University, 188 Shizi Street, Suzhou \\ 215000, China. Email: linchunyan1985@163.com.
}

Background: The outcomes for patients with acute myeloid leukemia (AML) have been shown to vastly differ, predominantly due to genetic heterogeneity. Cell adhesion molecules (CAMs) concluding numerous genes play an important role in AML. We aimed to systematically assess the expression characteristics of adhesion molecules and their correlation to the outcomes of AML.

Method: A total of 173 patients with AML were enrolled in this study. The genetic expressional information and clinical data sourced in previous studies were collected from the Cancer Genome Atlas (TCGA) database. The expression profiles of 141 CAMs were assessed, and the AML subgroups with specific patterns of expression were identified. The outcomes and clinical features of each AML subgroup were compared to detect the factors associated with prognosis. The differentially expressed genes (DEGs) between each subgroup were identified and the prognostic roles of those molecules were evaluated.

Results: According to subgroup clustering, both the primary cluster_1 and subcluster_1 showed a favorable prognosis compared to that of the other patients (26.3 vs. 17.0 months of overall survival (OS) and 46.5 vs. 15.8 months of OS, respectively). Both of the two subgroups were characterized by depressed human leukocyte antigen (HLA) genes. Assessment of the expression of prognosis-associated CAMs revealed that the expressions of SELE, NRCAM, ITGA4, and SDC1 were positively correlated with AML prognosis, while the expression of $L 1 C A M, P D C D 1, C D 276, S E L P L G$, and CLDN14 were negatively correlated with AML. Among the abovementioned genes, we detected that the individual gene expressions of NRCAM and VCAM1 were capable of independently predicting OS, and the OS was correlated with CAMs closely enough to enable the construction of models for prognosis prediction [area under the curve (AUC) $=0.78$ and AUC=0.77, respectively].

Conclusions: This study showed a landscape of the expression of CAMs in AML and identified a distinct subgroup with a significantly favorable prognosis. We detected that CAMs can assist in distinguishing the cohort with long term survival and constructed two models to predict the prognosis. Those CAMs have the potential to be developed as therapy targets in the treatment of AML.

Keywords: Acute myeloid leukemia (AML); cell adhesion molecules (CAMs); subgroup; prognosis

Submitted Nov 03, 2020. Accepted for publication Dec 15, 2020.

doi: $10.21037 /$ tcr-20-3315

View this article at: http://dx.doi.org/10.21037/tcr-20-3315 


\section{Introduction}

Acute myeloid leukemia (AML) is the most common malignant type of leukemia in adults, is associated with clonal hematopoietic stem-cell disorders, and has shown a disparate response to therapy (1). Although the majority of patients with newly diagnosed AML experience complete morphologic remission following treatment with intensive induction chemotherapy, the outcome in older patients who are unable to receive intensive chemotherapy without unacceptable side effects remains dismal $(1,2)$. Decisions about the choice of postremission therapy in patients with AML currently depend on the identification of a selected set of genetic markers at diagnosis and the detection of residual disease with multiparameter flow cytometry (3). Quantitative molecular evaluation during complete remission could further improve prognostication of outcomes in patients with AML (4). Emerging immunotherapies such as chimeric antigen receptor $T$ cells have advanced the treatment of acute lymphoblastic leukemia (5); so far, most of the targets have been membrane proteins and members of cell adhesion molecule (CAM) sets (6).

The CAMs are specific proteins, which expressed on the cell surface (7). They have been reported to play a critical role in multiple biologic processes, including hemostasis, the immune response, inflammation, embryogenesis, and development of neuronal tissue. There are four main groups: the integrin family, immunoglobulin superfamily, selectins, and cadherins. Membrane proteins that mediate immune cell-cell interactions fall into different categories, namely those involved in antigen recognition, costimulation, and cellular adhesion. Adhesion plays an important role both in normal hematopoiesis and in AML (8). Blasts of AML express many of the CAMs identified on normal hematopoietic precursors. The differential expression of CAMs between normal hematopoietic cells and leukemic blasts has been documented as differently expressed, likely reflecting the heterogeneity of the disease (9). A variety of processes within the bone marrow (BM) are governed by CAMs, including migration, homing, and quiescence. The AML blasts home to BM, as the CAM-mediated interaction with the niche protects them from chemotherapeutic agents. On the contrary, they then detach from the niches and move from the BM into the peripheral blood to colonize other sites such as the spleen and liver, possibly in a process that is reminiscent of epithelial-to-mesenchymal-transition in metastatic solid cancers (10). The expression of CAMs has a prognostic impact and there are ongoing efforts to therapeutically target adhesion in the fight against 50 leukemia (11).

In this study, we elucidate the transcriptional pattern of CAMs in a cohort of 173 patients with AML. All data collected from a precise published work and complete follow-up information were also available. We focused on the prognostic role of assembled genes and sole CAMs, and further sought the independent prognostic factors, which might play crucial roles in the development of AML and emerge as potential immunotherapy targets. We present the following article in accordance with the MADR reporting checklist (available at http://dx.doi. org/10.21037/tcr-20-3315).

\section{Methods}

\section{Patients and RNA-seq}

The normalized RNA sequencing data of 200 patients were obtained from a public database (cbioportal. org), and the corresponding clinical records were also collected (12). In the previously published study, patients underwent pathological diagnosis and detailed clinical features were recorded, including age, gender, and grade. All 200 patients were diagnosed with AML according to the histological records. Gene expressional values were shown as messenger (m)RNA z-score data and compared between each subject. Collectively, there were 27 cases missing related transcriptional information, and 173 eligible cases were enrolled in the final study. All public omics data sets used were generated by previous studies and ethical approval was granted prior to their use. The study was conducted in accordance with the Declaration of Helsinki (as revised in 2013).

\section{Bioinformatics}

Genes relevant to CAMs statistics annotated in the Kyoto Encyclopedia of Genes and Genomes (KEGG) database (kegg.jp/hsa04514) were enrolled in the current study (13). After excluding 8 genes lacking expressional information, the profiles of 141 genes involved in cell adhesion were assessed in AMLs. A cluster analysis of the genetic expression of integral gene sets was performed to distinguish samples based on gene expression profiles. Participants with similar gene expression patterns were identified from the entire population. The transcriptional levels were shown as mRNA z-scores and clustered using the hierarchical clustering algorithm via a Stanford 
program (14). The cluster heat map and pattern according to tumor stage were generated with the Java Treeview program (jtreeview.sourceforge.net) (15) and GraphPad Prism 8.0 (GraphPad Software, Inc., San Diego, CA, USA; Version 8).

\section{Prognostic relevance analyses}

The prognostic roles of the chromatin remodeling related genes were investigated by comparing the survivals of different groups. The overall survival (OS), progressionfree survival (PFS), disease-free survival (DFS), and diseasespecific survival (DSS) were accessed using a GraphPad Prism program (GraphPad Software, Inc., San Diego, CA, USA; Version 8). Comparisons of survival in different clusters revealed the relevance of gene expressional profiles and the prognosis. Additionally, an analysis of the difference in OS between the cohorts with low or high expression levels of individual genes was conducted using GraphPad Prism 8.0.

\section{Statistical analysis}

Survival curves of different groups were plotted and compared using the log-rank test in GraphPad Prism 8.0. Differences in gene expression levels between clusters were detected using analysis of variance (ANOVA). Correlations between variables were determined by regression analyses. All tests were performed with the statistical software SPSS 24.0 (IBM, Inc., Armonk, NY, USA). Statistical significance was detected when a $\mathrm{P}$ value was $<0.05$.

\section{Results}

\section{The expressional profile of CAMs was significantly associated with AML survival}

To investigate the specific AML subpopulation, patients were divided into different groups according to similar CAMs expressing patterns. Primarily, there were two clusters which showed different expression models (Figure 1A). Compared to the outcomes in cluster_2, cluster_1 showed a better OS with an inapparent difference (26.3 vs. 17.0 months, $\mathrm{P}=0.2522$ ); however, cluster_1 had a significantly prolonged PFS (34.1 vs. 13.8 months, $\mathrm{P}=0.0379$ ) (Figure $1 B$ ). We also detected detailed subgroups according to different expression of CAMs, and identified four subclusters in total (Figure 1A). Comparison of prognoses of those subclusters showed that subcluster_1 147 clearly displayed the best OS and PFS (Figure 1C, upper). 148 Furthermore, we compared the survivals of patients 149 in subcluster_1 with the others and detected a notably 150 favorable OS (46.5 vs. 15.8 months) and PFS (undefined 151 period vs. 13.9 months) in subcluster_1, and the differences 152 were significant $(\mathrm{P}=0.0421$ and $\mathrm{P}=0.0137$, respectively). 153

\section{The expressions of human leukocyte antigen $(H L A)$ were wildly varied in $A M L$}

After having identified specific AML cohorts with diverse prognoses, we assessed the differently expressed CAMs between each subgroup. There were 76 genes which showed discrepant expressional levels between cluster_1 and cluster_2, as shown in Figure 2A. Additionally, there were 67 genes showing discrepant expressional levels between subcluster_1 and other patients, as shown in Figure $2 B$. Comparison of the two differently expressed genes (DEGs) sets revealed 50 genes in the overlaps represented by HLAs. Multiple genes of HLAs members, such as HLA-DMB [DM beta chain] and HLA-DPA1 (DP alpha 1 chain) (Figure 2C), were significantly downregulated in both cluster_1 and subcluster_1 $(\mathrm{P}<0.05)$. On the other side, genes like NTNG2, SPN, CNTN1, NRXN3, $P V R$ and $C L C N 10$ were significantly highly-regulated either in cluster_1 or subcluster_1 $(\mathrm{P}<0.05)($ Table 1$)$.

\section{Expression of CAMs was correlated with AML prognosis}

We arranged the AML participants in the order of survival status and assessed the prognosis-correlated genes from the CAMs. Finally, 21 genes were detected to be significantly associated with the OS of AML $(r>0.15$ or $<-0.15, \mathrm{P}<0.05)$ (Figure 3A). Among those genes, expressions of 10 genes were positively correlated to OS (e.g., SELE and NRCAM); expressions of 9 genes were negatively correlated to OS (e.g., L1CAM and PDCD1) (Tables 2,3). We performed multifactor regression analysis in order to identify independently prognostic CAMs. In total, there were 5 genes, L1CAM, SDC1, NTNG1, CLDN14 and NRCAM, detected as independently correlated with OS. We compared the OS between the high expression subgroup and low expression subgroup regarding single genes. Among those genes, patients with up-regulated NRCAM showed a significantly prolonged OS comparing to the down-regulated cohort (27.0 vs. 11.8 months, $\mathrm{P}=0.0133$ ) (Table 4, Figure 3B). Upregulation of $S D C 1$ also indicated a favorable prognosis 


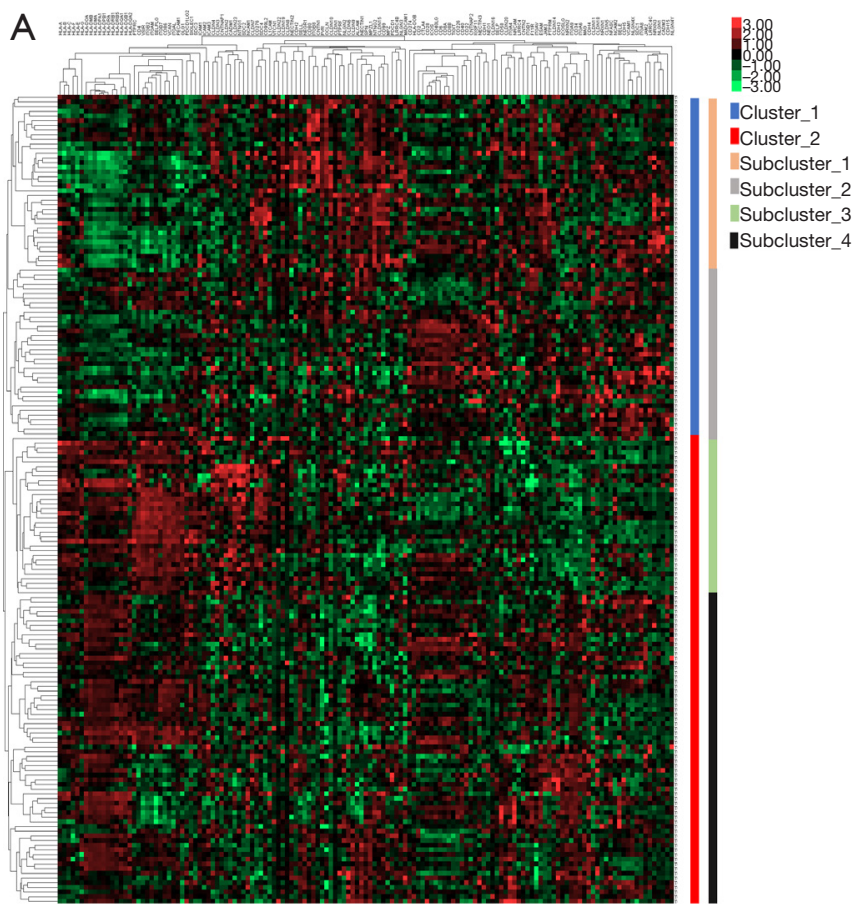

C

OS subcluster 1234

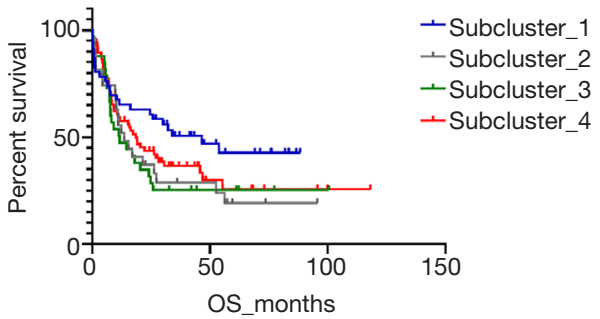

OS subcluster 1 vothers

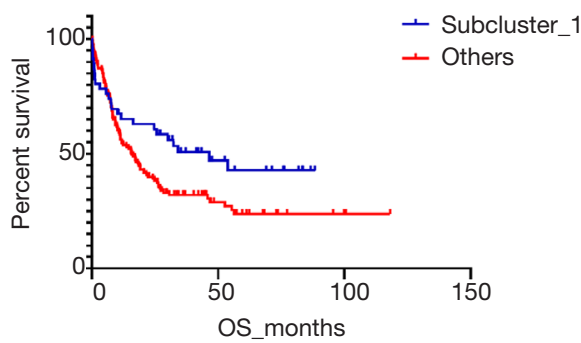

B

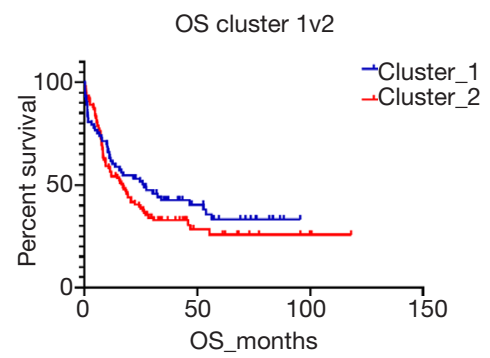

PFS cluster 1v2

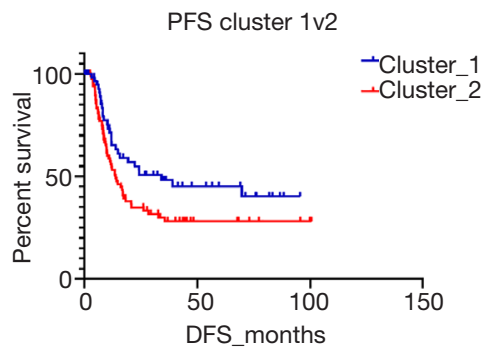

PFS subcluster 1234

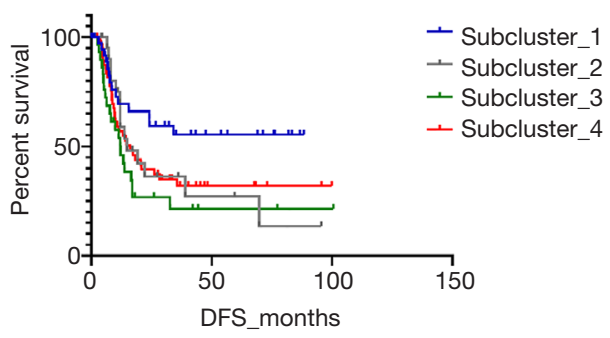

PFS subcluster 1vothers

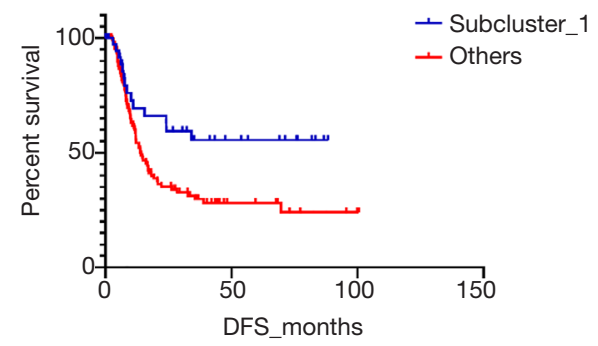

Figure 1 Analysis of CAMs expression showed two primary clusters and four subclusters in AML. (A) Hierarchical clustering divided the entire participant group into different subgroups; (B) the participants in cluster_1 showed a favorable OS and PFS; (C) the participants in subclusters showed different outcomes (Top) and subcluster_1 showed favorable OS and PFS compared to the others (Bottom). CAMs, cell adhesion molecules; AML, acute myeloid leukemia; OS, overall survival; PFS, progression-free survival. 
A

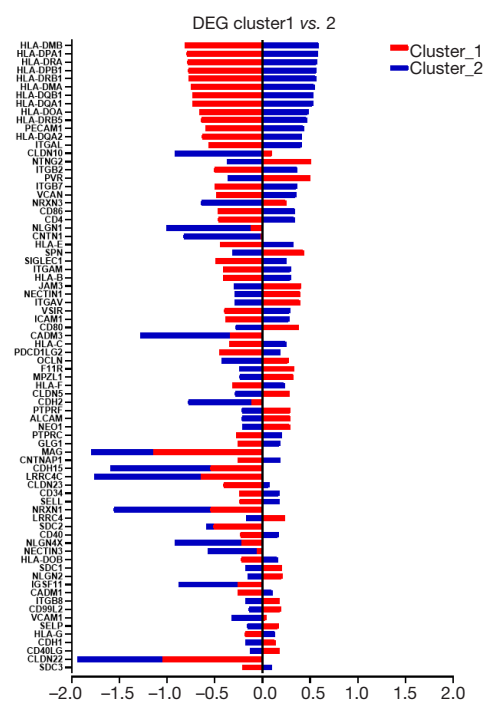

B

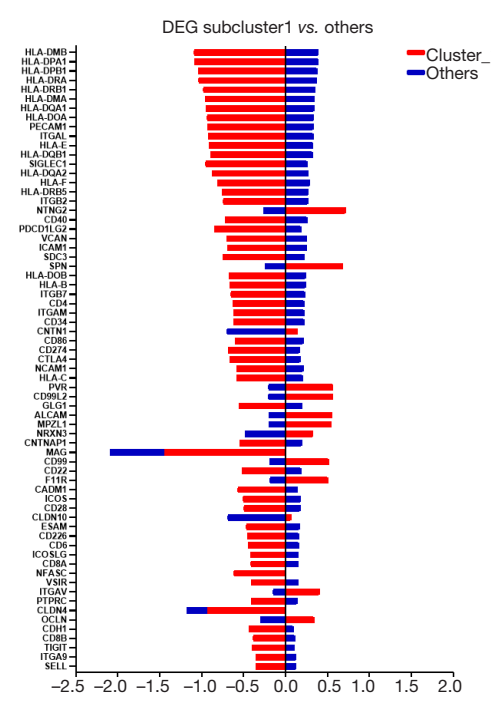

C

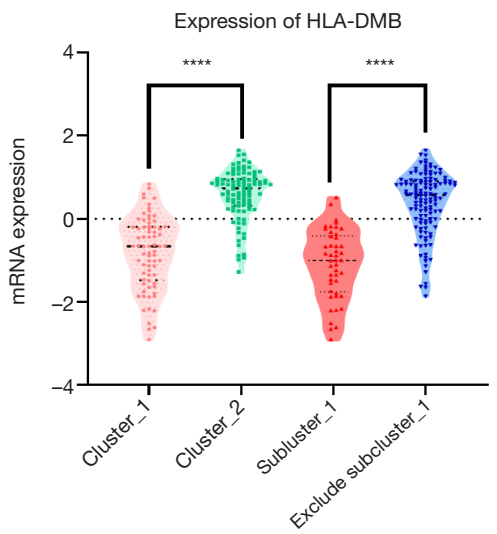

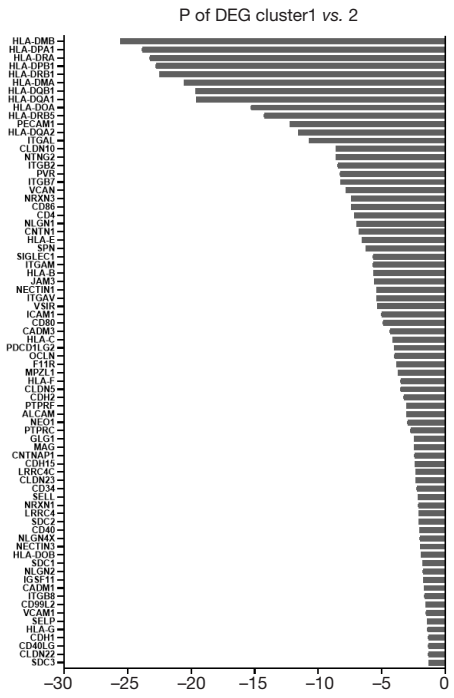

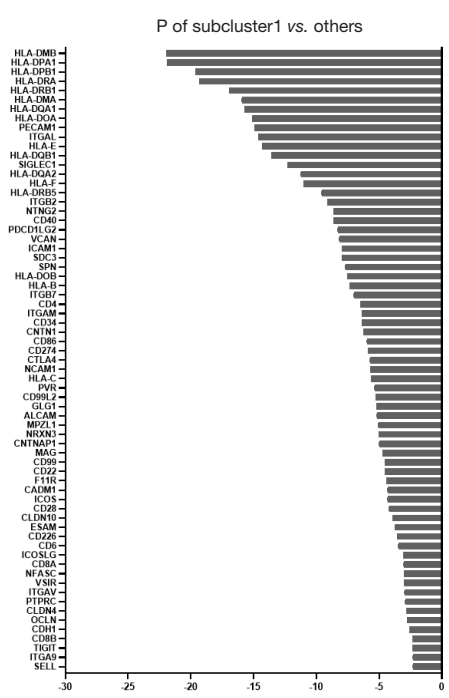

Expression of HLA-DPA1

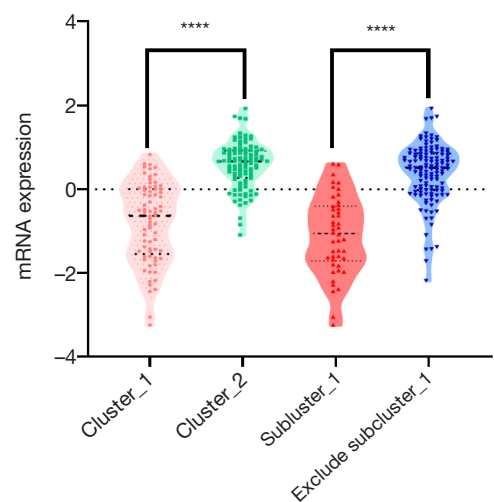

Figure 2 There were different expressions of CAMs in each subgroup. (A) The DEGs between cluster_1 and cluster_2; (B) the DEGs between subcluster_ 1 and the remaining participants; (C) the different expression of HLA-DMB and HLA-DPA1 in each cohort. CAMs, cell adhesion molecules; DEGs, differentially expressed genes; HLA, human leukocyte antigen. 
Table 1 The OS and PFS of clusters and subclusters

\begin{tabular}{|c|c|c|c|c|}
\hline Groups & OS (month) & $P$ value & PFS (month) & $P$ value \\
\hline Cluster_1 & 26.3 & 0.2522 & 34.1 & $0.0379^{*}$ \\
\hline Cluster_2 & 17.0 & & 13.8 & \\
\hline \multicolumn{5}{|l|}{ Subordinate } \\
\hline Others & 15.8 & & 13.9 & \\
\hline
\end{tabular}

${ }^{*} \mathrm{P}<0.05$. OS, overall survival; PFS, progression free survival.

A

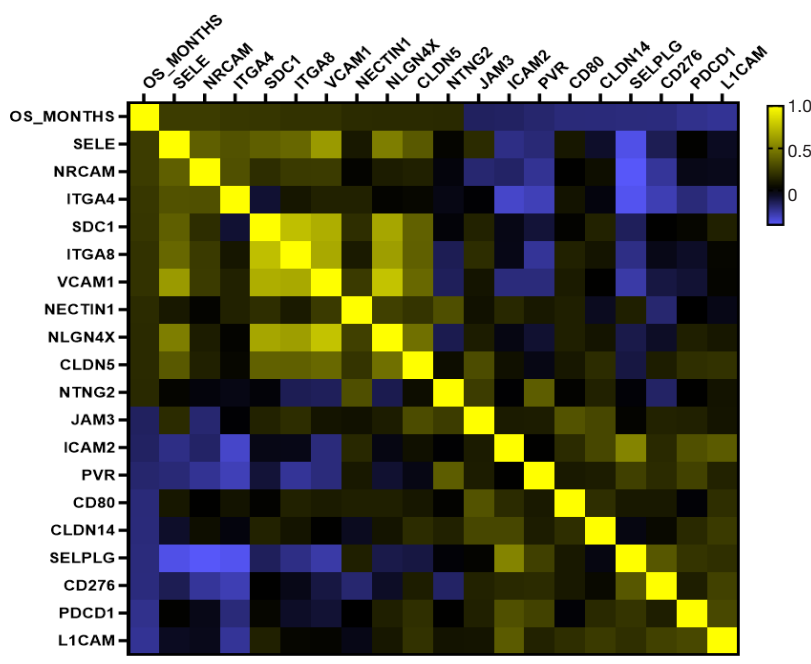

B

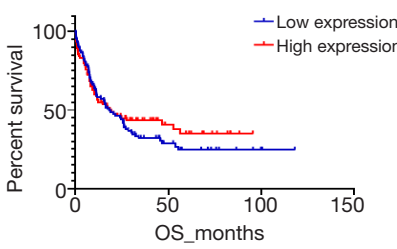

NTNG1

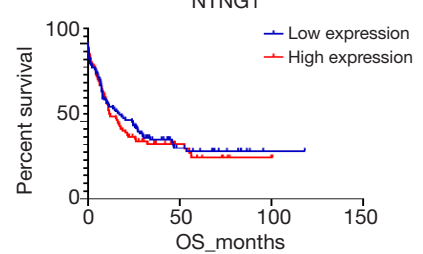

NRCAM

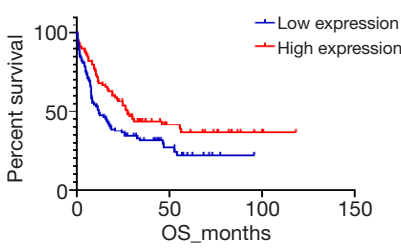

VCAM1

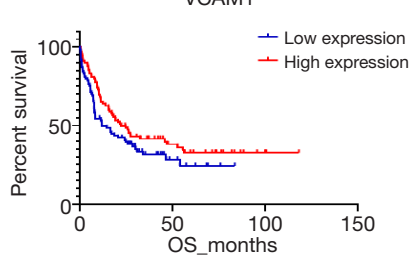

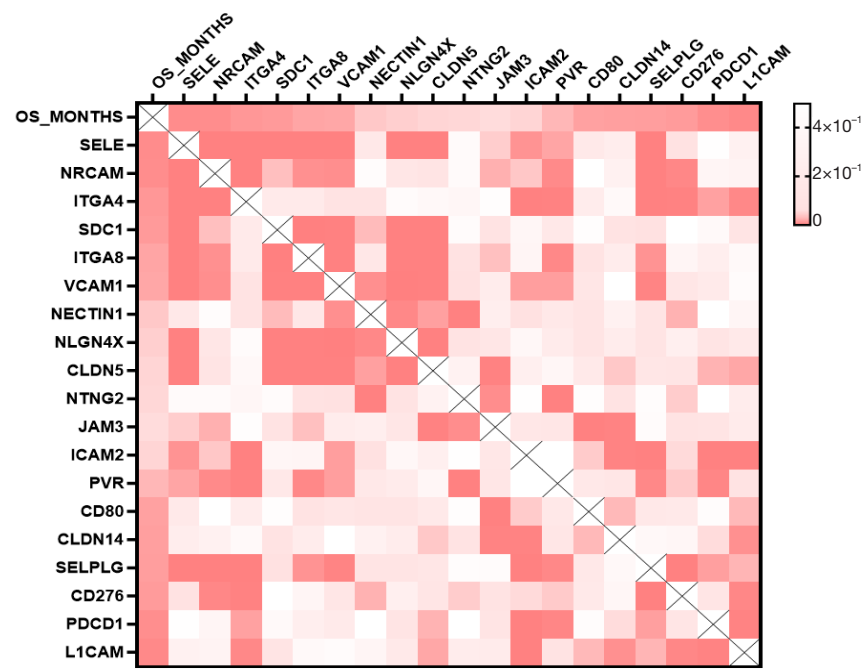

SDC1
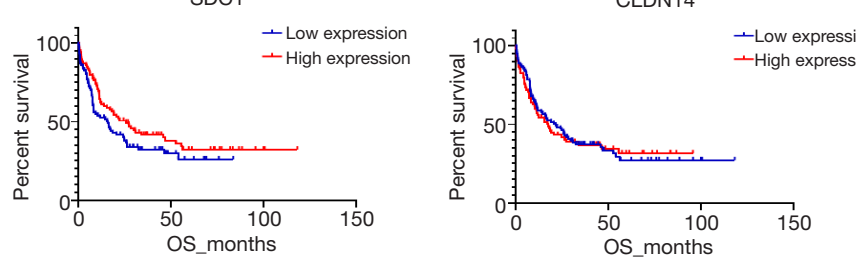

ITGA4

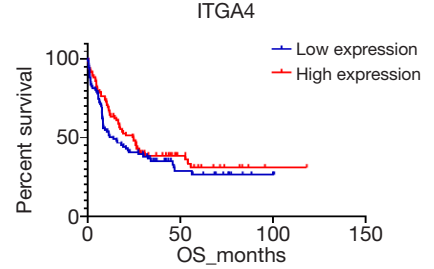

Figure 3 The expression of CAMs associated with AML OS. (A) The heatmaps of OS correlated genes (left: Pearson correlation, right: $\mathrm{P}$ value); (B) the different OS of participants with high and low expressed CAMs. CAMs, cell adhesion molecules; AML, acute myeloid leukemia; OS, overall survival. 
Table 2 The differentially expressed genes in subclusters

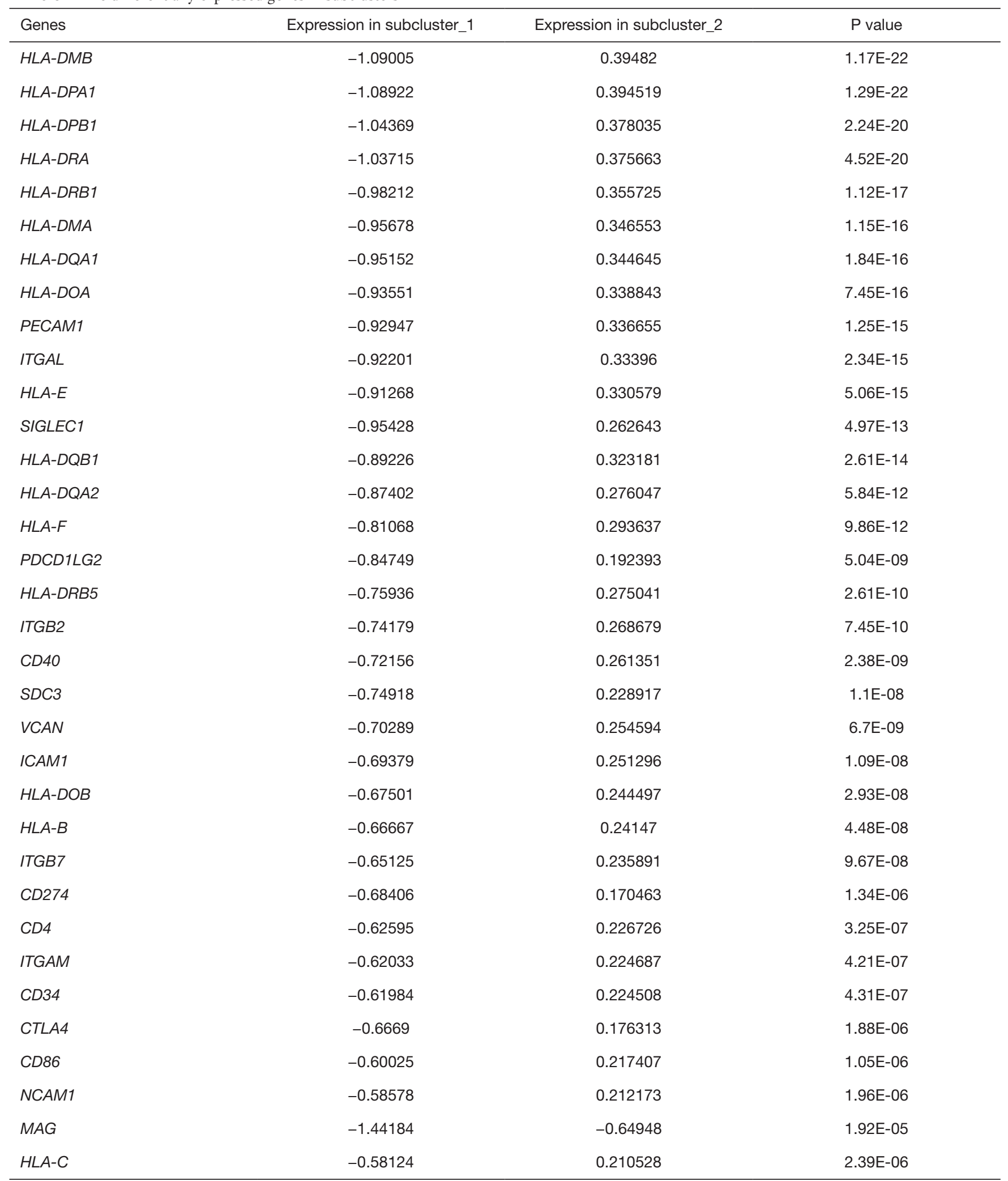

Table 2 (continued) 
Table 2 (continued)

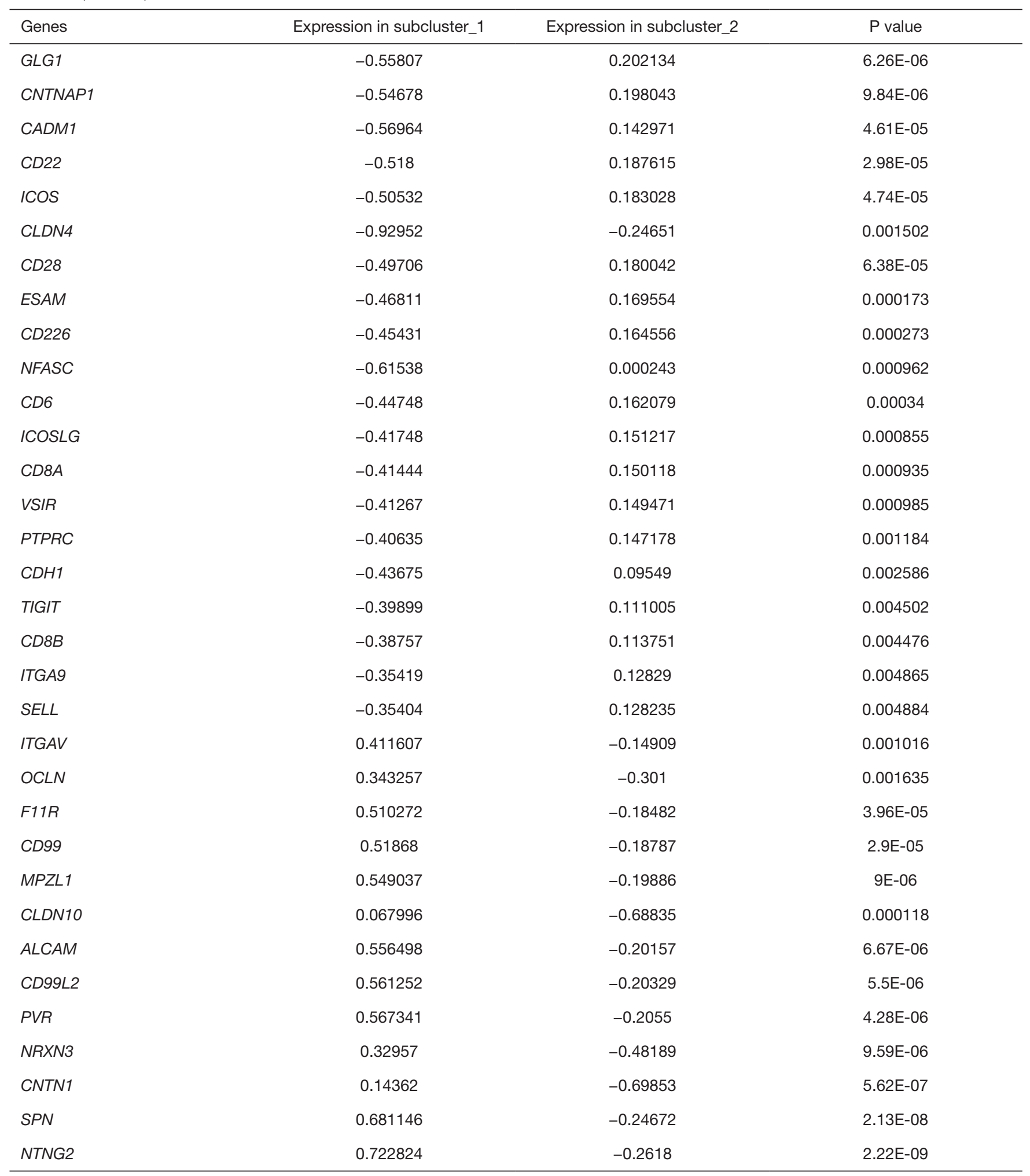


Table 3 The differentially expressed genes in clusters

\begin{tabular}{|c|c|c|c|}
\hline Genes & Expression in cluster_1 & Expression in cluster_2 & $\mathrm{P}$ value \\
\hline$H L A-D P A 1$ & -0.79287 & 0.578797 & $1.4 \mathrm{E}-24$ \\
\hline$H L A-D R A$ & -0.78532 & 0.573288 & $5.52 \mathrm{E}-24$ \\
\hline$H L A-D P B 1$ & -0.77898 & 0.568658 & 1.7E-23 \\
\hline$H L A-D Q B 1$ & -0.73524 & 0.536722 & $2.22 \mathrm{E}-20$ \\
\hline$H L A-D Q A 1$ & -0.73474 & 0.536355 & $2.4 \mathrm{E}-20$ \\
\hline$H L A-D O A$ & -0.66189 & 0.483176 & $5.21 \mathrm{E}-16$ \\
\hline HLA-DRB5 & -0.64205 & 0.46869 & $5.58 \mathrm{E}-15$ \\
\hline ITGAL & -0.56436 & 0.411991 & $1.85 \mathrm{E}-11$ \\
\hline ITGB2 & -0.50369 & 0.367694 & $3.43 \mathrm{E}-09$ \\
\hline ITGB7 & -0.49756 & 0.363226 & 5.54E-09 \\
\hline VCAN & -0.48534 & 0.354304 & $1.41 \mathrm{E}-08$ \\
\hline$C D 86$ & -0.47118 & 0.343958 & $4 \mathrm{E}-08$ \\
\hline$C D 4$ & -0.46371 & 0.338512 & $6.82 \mathrm{E}-08$ \\
\hline$H L A-E$ & -0.44415 & 0.324236 & $2.62 \mathrm{E}-07$ \\
\hline SIGLEC1 & -0.49211 & 0.253827 & 2.05E-06 \\
\hline$H L A-C$ & -0.34751 & 0.253686 & $7.28 \mathrm{E}-05$ \\
\hline$H L A-F$ & -0.31787 & 0.23205 & 0.000301 \\
\hline MAG & -1.14477 & -0.6524 & 0.003414 \\
\hline CLDN23 & -0.40655 & 0.076474 & 0.004711 \\
\hline PTPRC & -0.27473 & 0.200549 & 0.00188 \\
\hline GLG1 & -0.2595 & 0.189431 & 0.003372 \\
\hline CNTNAP1 & -0.25628 & 0.18708 & 0.0038 \\
\hline$S D C 2$ & -0.51325 & -0.07472 & 0.008187 \\
\hline CD34 & -0.24505 & 0.17889 & 0.005699 \\
\hline SELL & -0.2397 & 0.174983 & 0.006873 \\
\hline$C D 40$ & -0.23042 & 0.168201 & 0.00943 \\
\hline$H L A-D O B$ & -0.22227 & 0.162263 & 0.012329 \\
\hline CADM1 & -0.25654 & 0.106812 & 0.022345 \\
\hline
\end{tabular}

Table 3 (continued) 
Table 3 (continued)

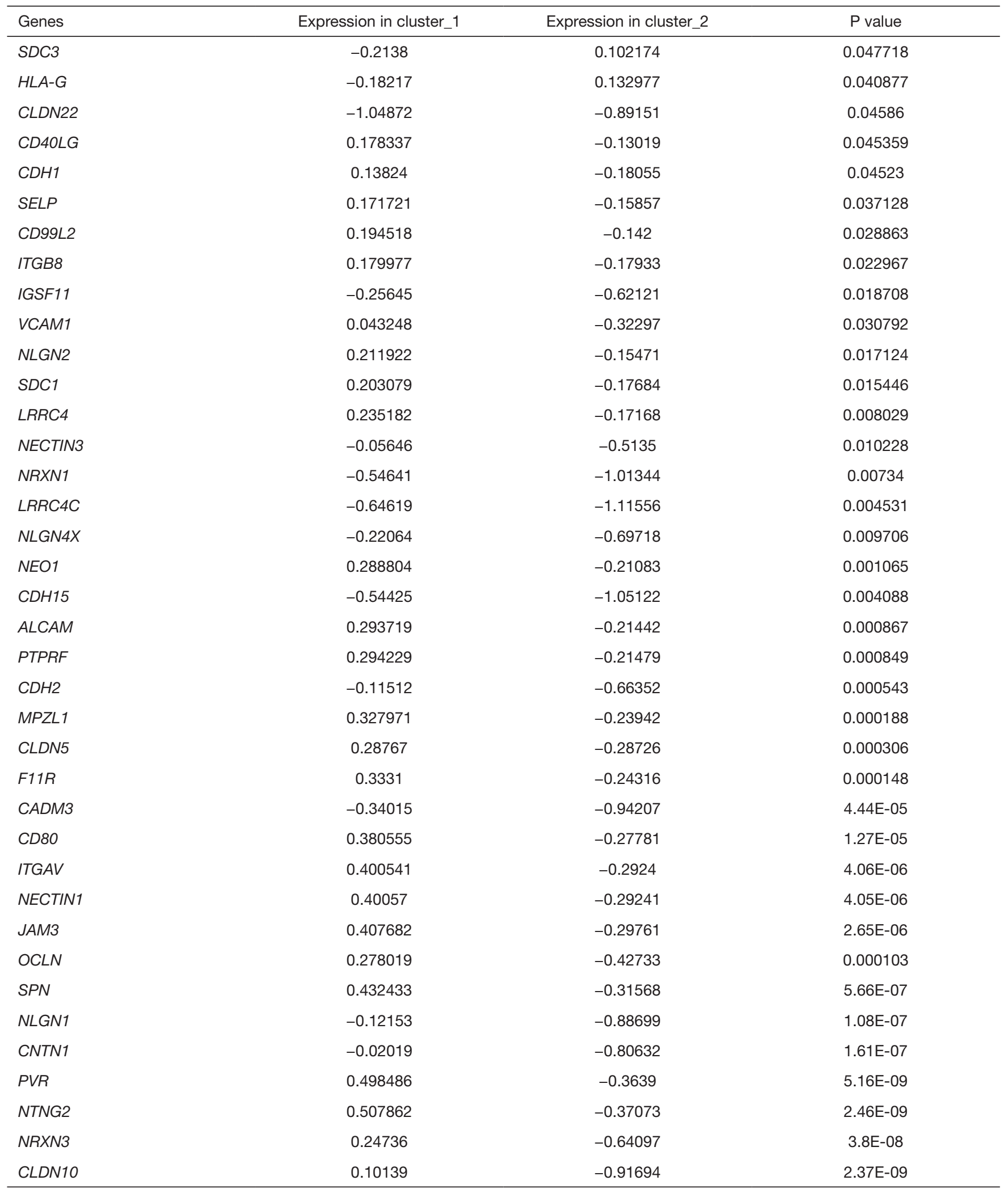


Table 4 The OS-correlated CAMs

\begin{tabular}{|c|c|c|}
\hline Genes & Pearson correlation & $P$ value \\
\hline$N R C A M$ & 0.233 & 0.006 \\
\hline ITGA4 & 0.214 & 0.011 \\
\hline$S D C 1$ & 0.208 & 0.014 \\
\hline VCAM1 & 0.194 & 0.020 \\
\hline NECTIN1 & 0.168 & 0.037 \\
\hline NLGN4X & 0.164 & 0.041 \\
\hline CLDN5 & 0.161 & 0.044 \\
\hline ICAM2 & -0.161 & 0.044 \\
\hline$P V R$ & -0.180 & 0.028 \\
\hline$C D 80$ & -0.199 & 0.017 \\
\hline CLDN14 & -0.202 & 0.016 \\
\hline SELPLG & -0.204 & 0.015 \\
\hline$C D 276$ & -0.207 & 0.014 \\
\hline$P D C D 1$ & -0.230 & 0.007 \\
\hline L1CAM & -0.244 & 0.005 \\
\hline
\end{tabular}

OS, overall survival; CAMs, cell adhesion molecules.

in AML, but only with a slight significance $(\mathrm{P}=0.0531)$ (Table 4, Figure 3B). Moreover, we divided the entire participant cohort into two groups, a good prognosis group and poor prognosis group, according to their relevant OSs. There were 3 genes, CLDN14, ITGA4, and VCAM1, that were significantly correlated to the assignment to these groups (Table 4, Figure 3B). Among them, up-regulation of $V C A M 1$ was notably correlated with favorable prognosis in AML (22.3 vs. 11.8 months, $\mathrm{P}=0.0449)$.

\section{The CAMs was capable of predicting OS}

According to the regression analysis, we identified genes that were independently correlated with OS and independently capable of distinguishing the participants into groups with good prognosis or poor prognosis (Table 5). To predict the AML OS, we determined the independent prognostic factors (CAMs) and constructed two OS prediction models. Both of the models had acceptable efficiency to predict the
OS (AUC $=0.78$ and 0.77, respectively) (Figure 4). Statistic 214 evaluation showed that the two models were significant in 215 projecting OS of patients with AML (Table 6). 216

Discussion $\quad 218$

In this study, we described the landscape of CAMs that 220 are expressed in AML. Cell adhesion is a process through 221 which cells interact with and attach to neighboring cells or 222 matrix using specialized surface CAMs. Adhesion plays an 223 important role in both normal hematopoiesis and AML. 224 Many of the AMs identified on normal hematopoietic 225 precursors are also expressed by AML blasts. Differential 226 expression of AMs between normal hematopoietic cells 227 and leukemic blasts has been documented as variable, likely 228 reflecting the heterogeneity of the disease. Prognosis is 229 affected by the expression of AMs and efforts continue to be 230 made to therapeutically target adhesion in the fight against 231 leukemia. 
Table 5 The prognostic roles of independent prediction genes

\begin{tabular}{|c|c|c|c|c|c|}
\hline Genes & Low expression & High expression & $P$ value & $\mathrm{HR}$ & $95 \% \mathrm{Cl}$ of ratio \\
\hline SDC1 & 15.8 & 25.8 & 0.0531 & & \\
\hline NTING1 & 24.1 & 16.4 & 0.6139 & & \\
\hline CLDN14 & 21.5 & 17.4 & 0.8518 & & \\
\hline VCAM1 & 11.8 & 22.3 & 0.0449 & 1.378 & $0.9464-2.005$ \\
\hline ITGA4 & 13.6 & 24.6 & 0.2778 & & \\
\hline
\end{tabular}

$\mathrm{Cl}$, confidence interval; $\mathrm{HR}$, hazard ratio.

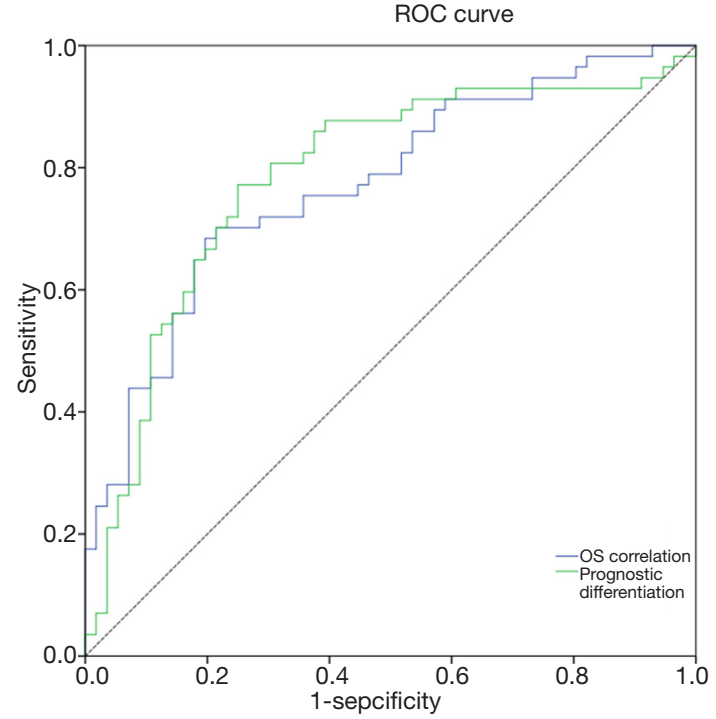

Figure 4 The ROC curves of two OS prediction models. ROC, receiver operating characteristic; OS, overall survival.
Different expression profiles revealed discrepant outcomes of AML. Both the primary cluster_1 (participants, $\mathrm{n}=73)$ and subcluster_1 ( $\mathrm{n}=46)$ showed significantly prolonged OS and PFS compared to the other groups. All the differences showed statistical significance $(\mathrm{P}<0.05)$ except the discrepancy in OS between cluster_1 and cluster_2 (26.3 vs. 17.0 months), which could be attributed to the limited subject size. Evaluation of the differently expressed CAMs indicated that the down-regulation of HLA-relevant genes was associated with a favorable prognosis in AML. Most of the prognosis-related HLAs belonged to major histocompatibility complex (MHC) class II antigen. Among those HLAs, HLA-DMB was shown as a representative molecule and reported to play a critical 246 role in the releasing of class II-associated invariant chain 247 peptide (CLIP) from newly synthesized MHC class II 248 molecules (16). A previous study showed that HLA- 249 DM expression on myeloid leukemic blasts correlated 250 with a poor outcome, which is concordant with our 251 finding (17). Another expression differential of MHC 252 class II genes, HLA-DPA1, was also significantly down- 253 regulated in the favorable prognosis groups (Figure 2C). 254 However, this result contrasts with a previous study, which 255 showed that the HLA-DPA1 expression was specifically 256 depressed in patients with relapse after transplantation or 257 chemotherapy (18). We speculated that this discordance 258 was caused by dynamic changes in the expression of HLAs in AML.

In the regression analysis for the detection of OScorrelated CAMs, neuronal cell adhesion molecule (NRCAM) was uncovered as an independent predictor and the expression was positively correlated with prolonged OS in AML. There is an involvement of NRCAM in the protein binding of heterotypic cell-cell adhesion, 266 and NRCAM was reported to be inhibited by miR- 267 29a and transcriptionally coactivated by Ski protein in 268 AML (19). Previous studies have also shown that NRCAM 269 is a potentially prognostic biomarker in solid tumors, such 270 as glioma (20) and gastric cancer (21). The vascular cell 271 adhesion molecule-1 (VCAM1) is involved in leukocyte- 272 endothelial cell adhesion and interacts with integrin 273 alpha-4/beta-1 (ITGA4/ITGB1) on leukocytes (22). In the 274 current study, VCAM1 was notably and positively correlated 275 with OS; moreover, up-regulation of VCAM1 was seen 276 to independently predict a favorable prognosis in AML. 277 Consistently, previous articles have reported that VCAM1 278 
Table 6 Two prediction models were constructed for indicating the prognosis of AML

\begin{tabular}{|c|c|c|c|c|c|c|c|}
\hline Models & Variables & Coefficient & AUC & $95 \% \mathrm{Cl}$ of $\mathrm{AUC}$ & Sensitivity & Specificity & $P$ value \\
\hline \multirow{3}{*}{ OS correlation } & ITGA4 & 0.673472 & \multirow{3}{*}{0.78} & \multirow{3}{*}{$0.70-0.87$} & \multirow{3}{*}{0.68} & \multirow{3}{*}{0.80} & \multirow{3}{*}{$1.79 \mathrm{E}-07$} \\
\hline & VCAM1 & 0.578583 & & & & & \\
\hline & Constant & -0.06208 & & & & & \\
\hline \multirow{4}{*}{$\begin{array}{l}\text { Prognostic } \\
\text { differentiation }\end{array}$} & SDC1 & 3.821098 & \multirow{4}{*}{0.77} & \multirow{4}{*}{$0.69-0.86$} & \multirow{4}{*}{0.77} & \multirow{4}{*}{0.75} & \multirow{4}{*}{$6.4 \mathrm{E}-07$} \\
\hline & NTNG1 & -3.01709 & & & & & \\
\hline & CLDN14 & -2.63531 & & & & & \\
\hline & NRCAM & 2.149581 & & & & & \\
\hline
\end{tabular}

$\mathrm{Cl}$, confidence interval; $\mathrm{AUC}$, area under the curve; OS, overall survival; AML, acute myeloid leukemia.

was more highly expressed on normal cells compared with leukemic bone marrow stromal cells. These findings suggest that the expression of NRCAM and VCAM1 are efficient predictive markers in AML.

Finally, based on the regression analysis, we constructed two prediction models for indicating the prognosis of AML. Both of the models showed a good efficiency and had AUCs of 0.78 and 0.77 , respectively. The results suggested that the RNA-seq data of CAMs have the potential to predict OS. However, since the study is limited to a retrospective data set and has not been verified in the prospective subjects, determining its prognostic roles of CAMs requires further exploration. The specific mechanisms of CAMs to promote or suppress the AML also need to be deeply investigated.

\section{Conclusions}

In conclusion, we used data obtained from a public database to retrospectively analyze the expression profiles of CAMs and the prognostic roles of sole genes of CAMs in 173 patients with AML. According to gene expression values, specific subgroups with favorable prognoses and independent OS prediction genes were identified. Prospective clinical studies are required for further validation of these results.

\section{Acknowledgments}

None.

\section{Footnote}

Reporting Checklist: The authors have completed the MADR reporting checklist. Available at http://dx.doi.org/10.21037/ tcr-20-3315

Conflicts of Interest: All authors have completed the ICMJE uniform disclosure form (available at http://dx.doi. org/10.21037/tcr-20-3315). The authors have no conflicts of interest to declare.

Ethical Statement: The authors are accountable for all aspects of the work in ensuring that questions related to the accuracy or integrity of any part of the work are appropriately investigated and resolved. All public omics data sets used were generated by previous studies and ethical approval was granted prior to their use. The study was conducted in accordance with the Declaration of Helsinki (as revised in 2013).

Open Access Statement: This is an Open Access article distributed in accordance with the Creative Commons Attribution-NonCommercial-NoDerivs 4.0 International License (CC BY-NC-ND 4.0), which permits the noncommercial replication and distribution of the article with the strict proviso that no changes or edits are made and the original work is properly cited (including links to both the formal publication through the relevant DOI and the license). See: https://creativecommons.org/licenses/by-nc$\mathrm{nd} / 4.0 \%$. 


\section{References}

1. Döhner H, Weisdorf DJ, Bloomfield CD. Acute Myeloid Leukemia. N Engl J Med 2015;373:1136-52.

2. Short NJ, Zhou S, Fu C, et al. Association of Measurable Residual Disease With Survival Outcomes in Patients With Acute Myeloid Leukemia: A Systematic Review and Meta-analysis. JAMA Oncol 2020;6:1890-9.

3. Papaemmanuil E, Gerstung $M$, Bullinger L, et al. Genomic Classification and Prognosis in Acute Myeloid Leukemia. N Engl J Med 2016;374:2209-21.

4. Xu M, Zhao XL, Zhu Y, et al. ND4 mutations are more prevalent in patients with acute myeloid leukemia of M2 morphology. Transl Cancer Res 2018;7:1064-71.

5. Willier S, Rothämel P, Hastreiter M, et al. CLEC12A and $\mathrm{CD} 33$ co-expression as preferential target on pediatric AML for combinatorial immunotherapy. Blood 2020. [Epub ahead of print].

6. Kupsa T, Horacek JM, Jebavy L. The role of adhesion molecules in acute myeloid leukemia and (hemato) oncology: a systematic review. Biomed Pap Med Fac Univ Palacky Olomouc Czech Repub 2015;159:1-11.

7. Gruszka AM, Valli D, Restelli C, et al. Adhesion Deregulation in Acute Myeloid Leukaemia. Cells 2019;8:66.

8. Zhang H, Nakauchi Y, Köhnke T, et al. Integrated analysis of patient samples identifies biomarkers for venetoclax efficacy and combination strategies in acute myeloid leukemia. Nat Cancer 2020;1:826-39.

9. Horiguchi H, Tsujimoto H, Shinomiya N, et al. A Potential Role of Adhesion Molecules on Lung Metastasis Enhanced by Local Inflammation. Anticancer Res 2020;40:6171-8.

10. Oellerich T, Oellerich MF, Engelke $M$, et al. $\beta 2$ integrinderived signals induce cell survival and proliferation of AML blasts by activating a Syk/STAT signaling axis. Blood 2013;121:3889-99, S1-66.

11. Bae MH, Oh SH, Park CJ, et al. VLA-4 and CXCR4 expression levels show contrasting prognostic impact (favorable and unfavorable, respectively) in acute myeloid leukemia. Ann Hematol 2015;94:1631-8.

12. Gao J, Aksoy BA, Dogrusoz U, et al. Integrative analysis of complex cancer genomics and clinical profiles using the cBioPortal. Sci Signal 2013;6:pl1.

13. Zhang Z, Yu H, Jiang S, et al. Evidence for Association of Cell Adhesion Molecules Pathway and NLGN1 Polymorphisms with Schizophrenia in Chinese Han Population. PLoS One 2015;10:e0144719.

14. de Hoon MJ, Imoto S, Nolan J, et al. Open source clustering software. Bioinformatics 2004;20:1453-4.

15. Saldanha AJ. Java Treeview--extensible visualization of microarray data. Bioinformatics 2004;20:3246-8.

16. Weber DA, Evavold BD, Jensen PE. Enhanced dissociation of HLA-DR-bound peptides in the presence of HLA-DM. Science 1996;274:618-20.

17. Chamuleau ME, Souwer Y, Van Ham SM, et al. Class IIassociated invariant chain peptide expression on myeloid leukemic blasts predicts poor clinical outcome. Cancer Res 2004;64:5546-50.

18. Christopher MJ, Petti AA, Rettig MP, et al. Immune Escape of Relapsed AML Cells after Allogeneic Transplantation. N Engl J Med 2018;379:2330-41.

19. Teichler S, Illmer T, Roemhild J, et al. MicroRNA29a regulates the expression of the nuclear oncogene Ski. Blood 2011;118:1899-902.

20. Hu G, Wang R, Wei B, et al. Prognostic Markers Identification in Glioma by Gene Expression Profile Analysis. J Comput Biol 2020;27:81-90.

21. Liu JB, Jian T, Yue C, et al. Chemo-resistant Gastric Cancer Associated Gene Expression Signature: Bioinformatics Analysis Based on Gene Expression Omnibus. Anticancer Res 2019;39:1689-98.

22. Becker PS, Kopecky KJ, Wilks AN, et al. Very late antigen-4 function of myeloblasts correlates with improved overall survival for patients with acute myeloid leukemia. Blood 2009;113:866-74.

(English Language Editor: J. Jones)
Cite this article as: Cheng J, Han J, Lin C. A comprehensive assessment of the prognostic role of cell adhesion molecules in acute myeloid leukemia. Transl Cancer Res 2020;9(12): 7605-7618. doi: 10.21037/tcr-20-3315 